\title{
Transcriptome analyses of a Chinese hazelnut species Corylus mandshurica
}

\author{
Hui Ma, Zhiqiang Lu, Bingbing Liu, Qiang Qiu and Jianquan Liu*
}

\begin{abstract}
Background: Corylus was renowned for its production of hazelnut and taxol. To understand the local adaptation of Chinese species and speed up breeding efforts in China, we analyzed the leaf transcriptome of Corylus mandshurica, which had a high tolerance to fungal infections and cold.

Results: A total of 12,255,030 clean pair-end reads were generated and then assembled into 37,846 Expressed Sequence Tag (EST) sequences. During functional annotation, 26,565 ESTs were annotated with Gene Ontology (GO) terms using Blast2go and 11,056 ESTs were grouped into the Kyoto Encyclopedia of Genes and Genomes (KEGG) pathways using KEGG Automatic Annotation Server (KAAS). We identified 45 ESTs that were homologous to enzymes and transcription factors responsible for taxol synthesis. The most differentiated orthologs between $C$. mandshurica and a European congener, C. avellana, were enriched in stress tolerance to fungal resistance and cold.
\end{abstract}

Conclusions: In this study, we detected a set of genes related to taxol synthesis in a taxol-producing angiosperm species for the first time and found a close relationship between most differentiated genes and different adaptations to fungal infection and cold in C. mandshurica and C. avellana. These findings provided tools to improve our understanding of local adaptation, genetic breeding and taxol production in hazelnut.

Keyword: Corylus mandshurica, Transcriptome, Adaptation, Divergence, Fungi/fungus, Cold/frigid, Taxol/paclitaxel

\section{Background}

Corylus is an important genus, both economically and ecologically, in China. There is currently more than 4 million acres of natural hazel groves in northeastern China alone [1]. Its nuts, rich in unsaturated fat and vitamins, are widely consumed. Its leaves are used by local farmers to feed domestic silkworm [2]. Its stocks have been successfully used for grafting Castanea henryi to improve chestnut production and quality [3] and have also been shown to be an ideal substitute for logs of Carpinus cordata in Ganoderma culture [4]. A part from its clear economic importance, Corylus plays an important role in soil and water conservation owing to its strong root system and contributes to the sustainability of forests in this region [2].

Corylus species are also important sources of taxol (also named as Paclitaxel), which is an effective yet relatively expensive medicine for treatment of breast, ovarian and lung cancer [5-7]. Taxol was originally

\footnotetext{
* Correspondence: liujq@nwipb.ac.cn

Molecular Ecology Group, State Key Laboratory of Grassland Agro-ecosystem, School of Life Sciences, Lanzhou University, Lanzhou, Gansu, China
}

isolated from the bark of Pacific yew [8] and then later found to be present in the yew genus Taxus [9]. It was initially believed to occur only in gymnosperms, but was recently identified in leaves and fruits of a hazelnut species (C. avellana) [10]. Further studies validated this finding by showing that in vitro hazel cell cultures produce taxol and taxanes, indicating that it is not exclusively produced by symbiotic fungi [11-13]. Taxol was recently discovered in another hazelnut species, C. mandshurica (synonym to C. sieboldiana) [14]. However, except for the Corylus species as well as a few other species like Magujreothamnus speciosus, Morinda citrifolia, Justicia gendarussa and Yunnanopilia longistaminata [15,16], few angiosperm species have been reported to contain taxol or its derivatives. Interests in taxol production from hazel trees, especially from its leaves, have grown rapidly with the aim of conserving endangered yew species [17].

C. mandshurica is widely distributed in northeastern China and its nuts are characterized by a thin husk and high shelling percentage [18]. The nuts from this species are of higher quality in flavor and taste, and therefore command a higher price than the nuts from C. avellana. 
Moreover, C. mandshurica is highly resistant to Eastern Filbert Blight [19], a fungus that causes seriously damage to most commercially grown cultivars of $C$. avellana in the US [20], and has exceptional cold resistance; it is able to survive a frigid winter of $-48^{\circ} \mathrm{C}[21,22]$. All these traits make it a very desirable target for developing improved selections and breeding material [18,23]. Interspecific crossing and breeding experiments have been attempted between C. mandshurica and the commercial species C. avellana [1,22-26]. Molecular breeding aided by microsatellite marking has also been reported [27,28].

Next generation sequencing is a quick and cost-effective method for surveying the complete coding sequence of a genome. Much progress has been made in obtaining longer sequence reads, and many tools and algorithms have been developed to allow assembly of short reads. Despite the ever-increasing sequencing data, the Expressed Sequence Tags (ESTs) from C. avellana have only recently been released [29] and remain the only available large-scale sequencing data for the Corylus genus. In this study, we sequenced the leaf transcriptome of $C$. mandshurica native to China. Our aims were (1) to explore how homologous genes of two hazel species have differentiated to give their contrasting adaptations, and (2) to identify the possible genetic basis of taxol production in the genus Corylus by transcriptome assembly and gene annotation.

\section{Results and discussion}

\section{Sequencing and assembly}

After strict quality control, 12,255,030 clean pair-end reads were assembled into 37, 846 ESTs longer than 200 bp using Trinity [30]. The contig N50 was 799 bp and 8,328 ESTs had longer sequences. A total of 37,652 coding DNA sequences (CDSs) were predicted to have an average length of $431 \mathrm{bp}$ using Orfpredictor [31]. Comparison of our assembly with the Jefferson transcriptome assembly on C. avellana was shown in Table 1. It could be seen that the contig N50 of our assembly was slightly lower, which was partly due to the large increase in the number of assembled sequences. It was apparent from EST length distribution (Figure 1) that our assembly had more sequences at all length intervals beyond $160 \mathrm{bp}$. The assembled EST sequences of
C. mandshurica in fasta format were available in Additional file 1.

Given the recently released genome of Betula nana [32], we used BLAT [33] to map our transcriptome assembly against this genome that currently consisted of 551,915 contigs. We found that 32,078 ESTs mapped to 32,849 contigs. In comparison, 25,073 ESTs out of the Jefferson transcriptome assembly mapped to 25,841 contigs, with 19,908 contigs shared between the two hazelnut transcriptome assemblies (Figure 2). The ESTs that mapped to unique contigs might represent different genes specifically expressed in each species or different fragments of the same genes due to the fragmentary nature of the current Betula genome and the limited sequencing depth of the transcriptomes. Thus, our transcriptome analysis revealed many novel EST sequences for the Corylus genus that could not be identified from the Jefferson transcriptome assembly and helped locate the genomic locus for each EST, which had important implications for the development of further breeding markers of the Corylus species.

\section{Functional annotation}

To functionally classify the assembled ESTs, a homologybased approach was adopted in transcriptome annotation. A total of 30,536 ESTs gave hits on performing BLASTX searches [34] in the NCBI non-redundant protein database using an E-value cutoff of 1e-5, accounting for $80.7 \%$ of all assembled sequences. When sorting the top blast hits by species, Vitis vinifera was ranked first with 10,321 top blast hits, followed by Populus trichocarpa and Ricinus communis with 5,537 and 5,155 top blast hits, respectively (Figure 3). In addition, 26,565 ESTs were annotated with Gene Ontology (GO) terms using Blast2go [35] and 11,056 ESTs were annotated into Kyoto Encyclopedia of Genes and Genomes (KEGG) pathways with KEGG Automatic Annotation Server (KAAS) [36] using the Single-directional Best Hit (SBH) method.

\section{Identification of highly differentiated genes in the transcriptomes of C. mandshurica and C. avellana}

Since C. mandshurica and C. avellana were closely related species but with contrasting adaptations, our first goal was to identify which genes were highly differentiated.

Table 1 Comparison of transcriptome assembly and coding sequence prediction for Corylus mandshurica and Corylus avellana

\begin{tabular}{ccccc}
\hline & \multicolumn{2}{c}{ EST } & & CDS \\
\cline { 2 - 4 } & C. mandshurica & C. avellana & C. mandshurica & C. avellana \\
\hline Average Length & 580 & 532 & 831 & 377 \\
Length Range & $201 \sim 6821$ & 282549 & $30 \sim 4890$ & $42 \sim 4143$ \\
Numbers & 37846 & 961 & 37652 & 28167 \\
N50 Length & 799 & 4991 & 594 & 651 \\
Sequences (longer than N50) & 8328 & 8028 & 4945 \\
\hline
\end{tabular}




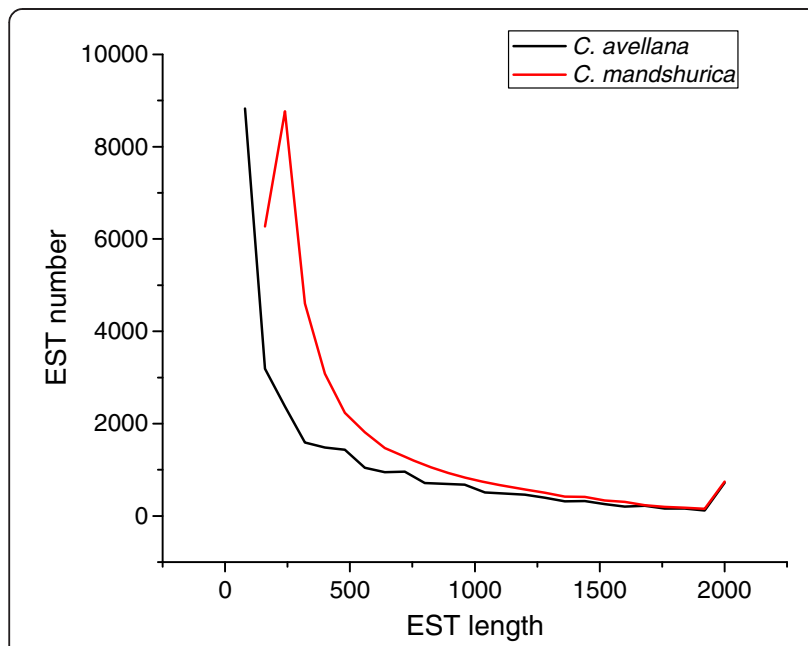

Figure 1 The length distribution of assembled ESTs from transcriptomes of $C$. mandshurica and C. avellana. ESTs are counted at an interval of $80 \mathrm{bp}$, with ESTs longer than $2000 \mathrm{bp}$ counted as $2000 \mathrm{bp}$. It is clear that more ESTs are assembled in the transcriptome of C. mandshurica than C. avellana at length intervals after $160 \mathrm{bp}$. (Trinity has a minimum length of $200 \mathrm{bp}$ in transcriptome assembly).

Using the available EST sequences for these two species, we performed a reciprocal blast to obtain best hit orthologs and compared both the sequence identities and presence of Insertion/Deletion (INDEL) according to BLASTN outputs. Since $98.7 \%$ of orthologs showed a sequence identity higher than $90 \%$ (Figure 4), we set a sequence identity of $90 \%$ as the low threshold in ortholog validation to exclude the presence of distantly related

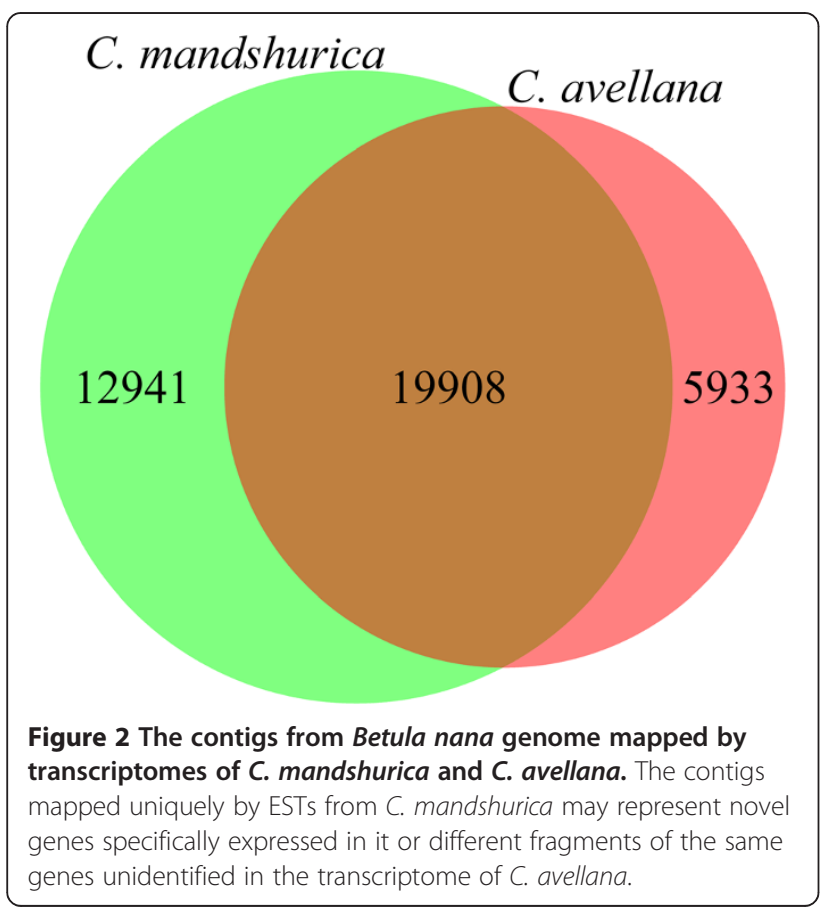

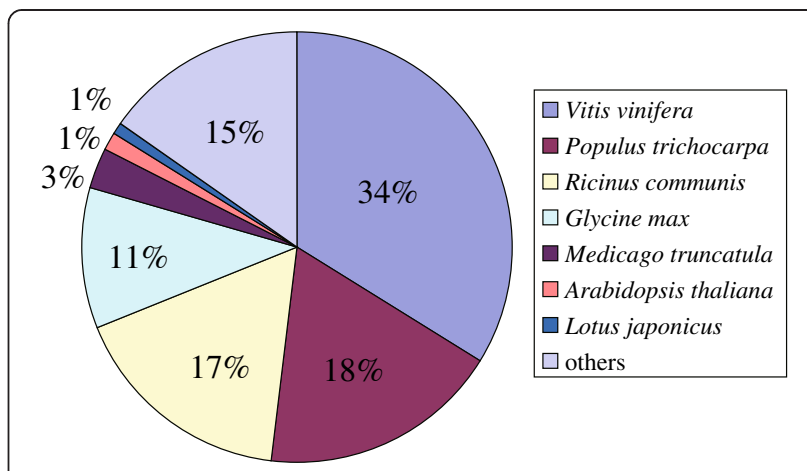

Figure $\mathbf{3}$ The percentage of top blast hits by species.

homologs. Because we were interested in orthologs with relatively great divergence between $C$. mandshurica and C. avellana, we took orthologs with the low sequence identity (less than 97\%), which account for $10.4 \%$ of all orthologs, as the highly differentiated genes. Furthermore, INDEL might cause different reading frames in coding regions of the two sets of orthologous ESTs [37]. It might also cause mRNA secondary structure change [38] in the coding and noncoding regions with alternative roles in transcriptional polyadenylation site selection [39], pre-mRNA splicing [40], mRNA stability, translation efficiency and protein folding [41,42]. Therefore, INDEL was also used as an indicator for sequence divergence. Thus, orthologs with gaps in the BLAST alignment were taken as another set of highly differentiated genes. Next, we performed separate GO enrichment analyses on these two types of differentiated orthologs using the WEGO web server [43]. GO terms for all orthologs and the two

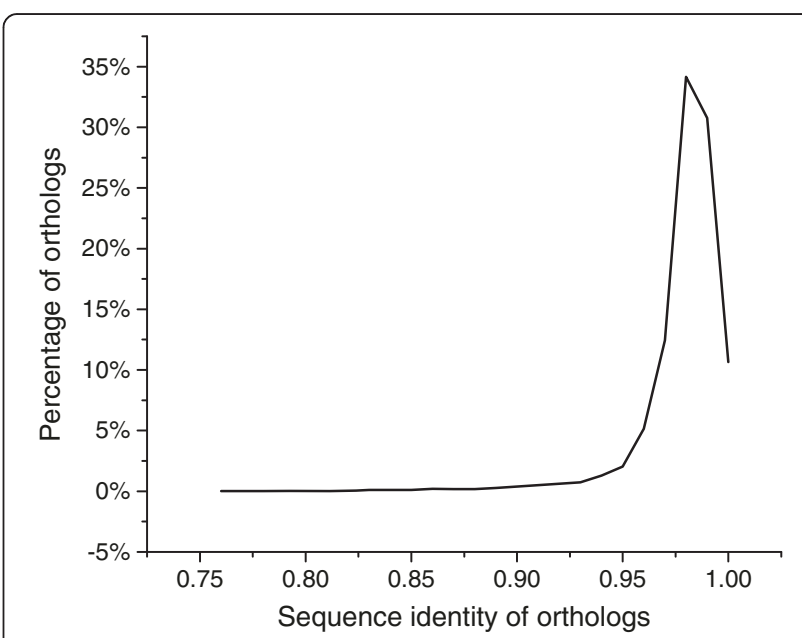

Figure 4 The distribution of sequence identity in blast regions for all orthologs. $98.7 \%$ orthologs have sequence identity no less than $90 \% ; 87.9 \%$ orthologs have sequence identity no less than $97 \%$. To exclude distantly homologous sequences, orthologs with sequence identity less than $90 \%$ are discarded in GO enrichment analysis. 


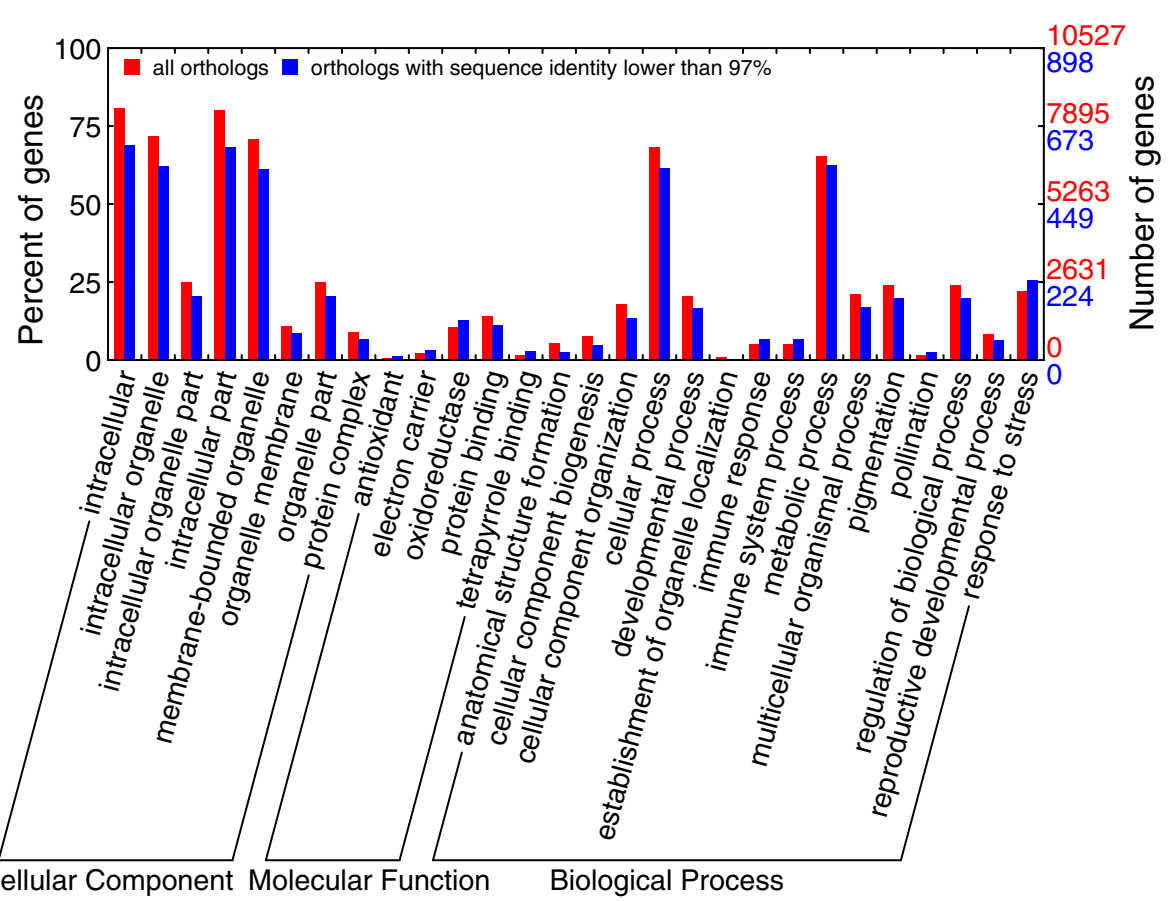

Figure $5 \mathrm{GO}$ enrichment on orthologs with sequence identity lower than $\mathbf{9 7 \%}$. Direct parent GO terms in biological process domain are displayed for simplicity. The GO terms in the biological process domain that show increase in ESTs with lower sequence identity are related to immune response and response to stress.

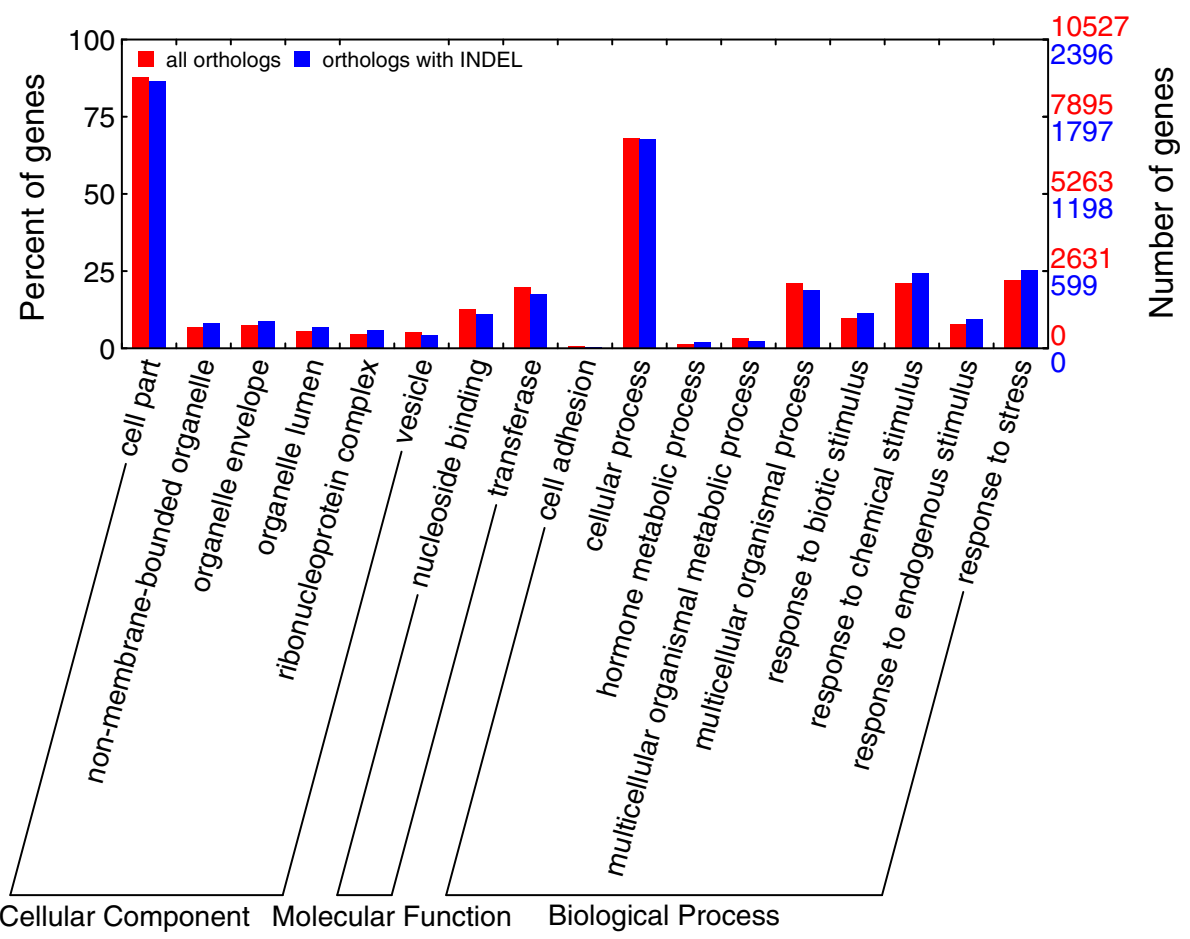

Figure 6 GO enrichment on orthologs with INDEL. Direct parent GO terms in biological process domain are displayed for simplicity. The GO terms in the biological process domain that show increase in ESTs with INDEL are related to hormone metabolic process and response to various stimuli (including response to stress). 
types of highly differentiated orthologs were available in Additional files 2, 3, 4).

According to the GO enrichment analyses (Figures 5 and 6), orthologs from most statistically significant GO terms were conserved in the respective sequences as they generally contained a low percentage of orthologs with a sequence identity lower than $97 \%$ or orthologs with INDEL. The conserved GO categories comprised GO terms in cellular process, developmental process and metabolic process (except hormone metabolic process) in the biological process domain for both species. All these processes were essential for plant survival. The divergent GO categories comprised immune, pollination and response to stress in the biological process domain for the GO enrichment of orthologs with sequence identities lower than 97\%. The orthologs with INDEL were enriched in hormone-related or various stimuli-related GO terms in the biological process domain (including hormone metabolic process and responses to various stimuli, especially response to stress). These findings suggested that C. mandshurica and C. avellana had become genetically differentiated whilst adapting to their different habitats.
Stress response genes were more prone to both sequence substitution and insertion/deletion, with occurrences of $25.6 \%$ and $25.2 \%$ among all differentiated ESTs, respectively. A close examination of GO terms under response to stress (Figures 7 and 8) revealed that three main categories displayed increased sequence divergence, including genes participated in defenses to bacteria and fungi, genes involved in cold tolerance and genes related to salt/drought/water stress. As C. mandshurica has better adapted to fungal infection and cold stress than C. avellana, further study of the highly divergent genes in C. mandshurica could identify the key genes responsible for the resistance to fungal infection and cold pressure. However, because orthologs were not necessarily one to one match between two species when gene duplication occurred after speciation, the identified orthologs could be either true alleles or different copies of the same family in the genomes. Under the latter scenario, differential expressions of the genes at both time and space should be carefully examined, which might also represent one of the adaptation mechanisms in this species.

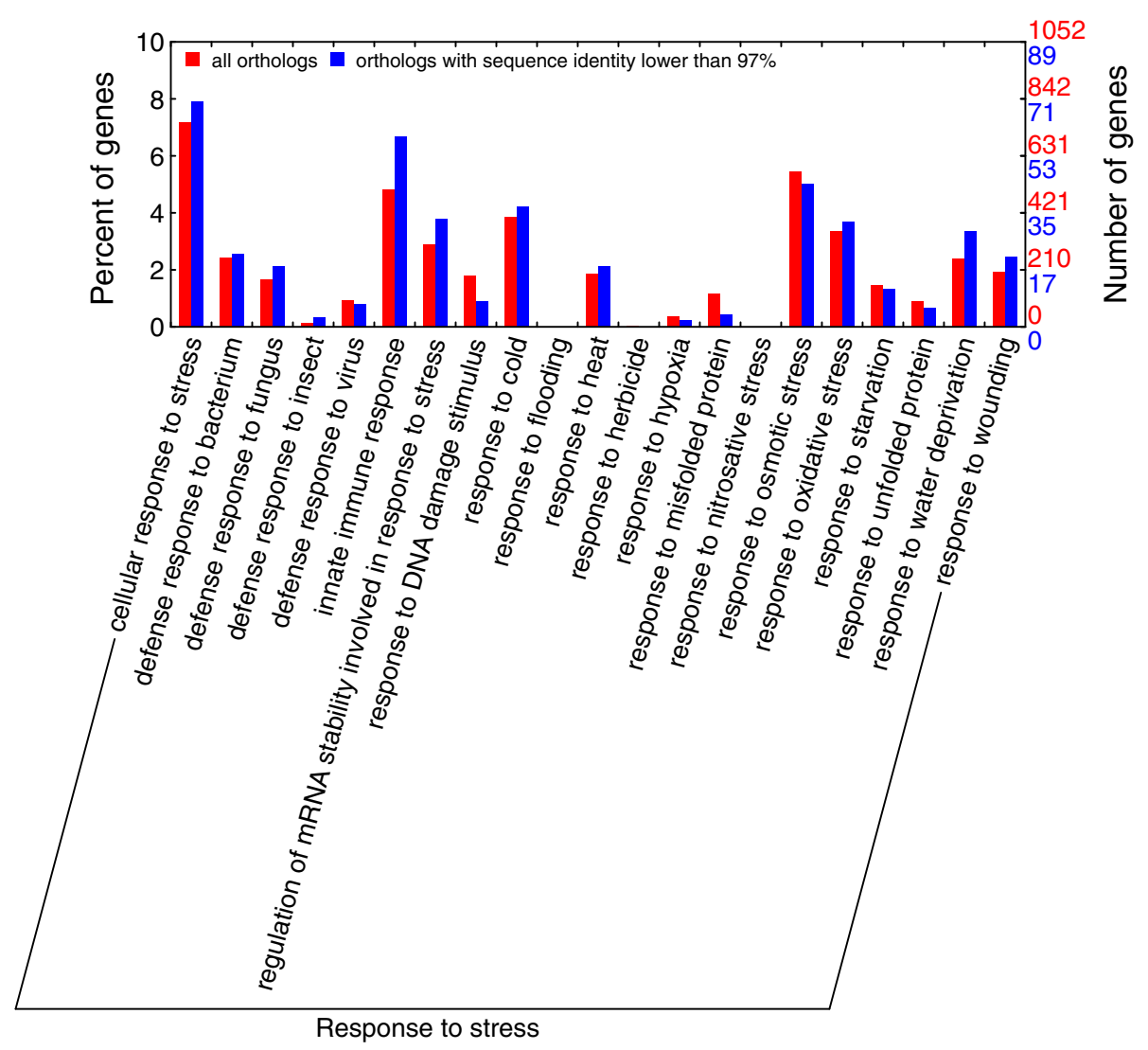

Figure $7 \mathrm{GO}$ terms under response to stress in GO enrichment on orthologs with low sequence identity. GO terms related to defense against bacteria and fungi (including innate immune response), tolerance to cold and heat, and salt/drought/water stress show increase in ESTs with lower sequence identity. 


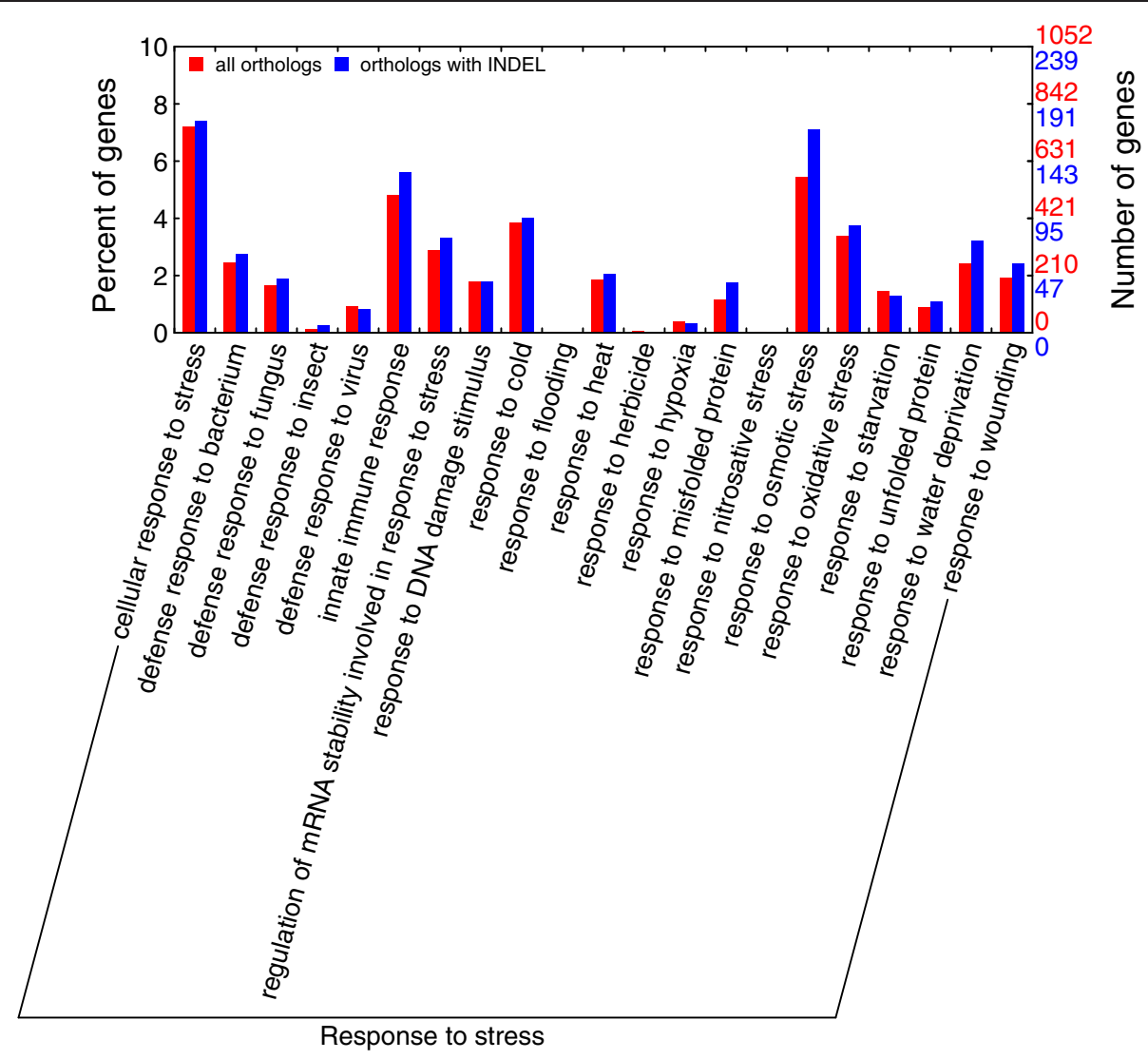

Figure $8 \mathrm{GO}$ terms under response to stress in GO enrichment on orthologs with INDEL. GO terms related to defense against bacteria and fungi (including innate immune response), tolerance to cold and heat, and salt/drought/water stress show increase in ESTs with INDEL.

\section{Genes responsible for taxol synthesis}

According to KEGG annotation, 29 ESTs were found to be involved in the terpene synthesis pathway. These included genes involved in isopentenyl-PP (IPP) synthesis in both the mevalonate and MEP/DOXP pathways and genes responsible for geranyl-PP and geranyl-geranyl-PP (GGPP) synthesis. The committing step for taxol production was the conversion of GGPP to taxa-4(5)-11(12)-diene in the diterpenoid biosynthesis pathway; however, genes involved in this reaction, as well as the following processes, were absent from our KEGG annotation. This was also encountered in the KEGG annotation of C. avellana transcriptome. Nonetheless, 31 ESTs (Table 2) were found to be homologous to the prototype genes participating in taxol synthesis in yew species, with sequence identities ranging from $23.93 \%$ to $50.32 \%$. This was similar to the sequence identities of $40 \% \sim 44.1 \%$ reported in some taxol-producing fungi [44] and was close to the maximal sequence identity of around $40 \% \sim 49.3 \%$ found between these genes and the available proteins from other plant species in the NCBI non redundant protein database (Table 3). In addition, 6 ESTs were found to be homologous to WRKY, and 8 ESTs homologous to JAMYC. These two transcriptional factors had been reported to induce taxol synthesis $[45,46]$.
Overall, our study reported for the first time large-scale identification of genes involved in the terpenoid pathway in Corylus, which would facilitate understanding of taxol synthesis in angiosperms, although further experiments were required to clarify the roles of these genes in such processes. On the other hand, it should be noted that not all sequences of the genes related to taxol synthesis were revealed by the present transcriptome analyses because of difficulties in normalizing all cDNAs before sequencing when the level of leaf mRNA expression in the taxol synthesis pathway was very low. In addition, some taxadiene synthase genes might only be expressed in response to external stimuli, such as naturally occurring fungal infection or artificial chemical induction [47]. Such genes would not be detected by the present approach. Since the family of terpene synthases were highly diversified across plants [48], it would be interesting to investigate the reasons why taxol production was shared by these special gymnosperm and angiosperm plants. Horizontal gene transfer was a likely cause of such convergent evolutions via symbiotic organisms. For example, three genes from different taxol-producing fungi (two from Ozonium sp. BT2 and one from Cladosporium cladosporioides) isolated from the inner tree barks [49-51] had been shown high sequence 
Table 2 ESTs homologous to genes involved in taxol synthesis in Taxus

\begin{tabular}{|c|c|c|c|c|}
\hline EST ID & Hit Protein GI & Identity (\%) & Length & Description \\
\hline comp37211_c1_seq1 & 386304248 & 36.51 & 189 & 10-deacetylbaccatin III-10-O-acetyl transferase, partial \\
\hline comp42386_c0_seq1 & 28558088 & 28.85 & 104 & 3'-N-debenzoyl-2'-deoxytaxol N-benzoyltransferase \\
\hline comp70594_c0_seq1 & 339521621 & 23.93 & 422 & C-13 phenylpropanoid side chain CoA acyltransferase \\
\hline comp70669_c0_seq1 & 28380187 & 34.95 & 432 & taxa-4(20),11(12)-dien-5alpha-ol-O-acetyltransferase \\
\hline comp68118_c0_seq1 & 28380187 & 28.44 & 450 & taxa-4(20),11(12)-dien-5alpha-ol-O-acetyltransferase \\
\hline comp53308_c0_seq1 & 386304662 & 38.04 & 163 & taxadienol acetyl transferase, partial \\
\hline comp119236_c0_seq1 & 53690152 & 45.98 & 87 & taxadien-5-alpha-ol-O-acetyltransferase \\
\hline comp68580_c0_seq1 & 53690152 & 30.06 & 173 & taxadien-5-alpha-ol-O-acetyltransferase \\
\hline comp37211_c0_seq1 & 53690152 & 32.39 & 142 & taxadien-5-alpha-ol-O-acetyltransferase \\
\hline comp83331_c0_seq1 & 53690152 & 28.84 & 215 & taxadien-5-alpha-ol-O-acetyltransferase \\
\hline comp64789_c0_seq1 & 53759170 & 42.6 & 446 & taxadiene 5-alpha hydroxylase \\
\hline comp57975_c0_seq1 & 386304485 & 50.32 & 155 & taxadiene 5nalpha hydroxylase, partial \\
\hline comp172528_c0_seq1 & 38201489 & 36.47 & 85 & taxa-4(5),11(12)-diene synthase \\
\hline comp193967_c0_seq1 & 15080743 & 46.03 & 63 & taxadiene synthase \\
\hline comp63152_c1_seq1 & 386304920 & 29.69 & 128 & taxadiene synthase, partial \\
\hline comp40035_c0_seq1 & 24266823 & 47.89 & 71 & 5-alpha-taxadienol-10-beta-hydroxylase \\
\hline comp53405_c1_seq1 & 24266823 & 49.47 & 95 & 5-alpha-taxadienol-10-beta-hydroxylase \\
\hline comp133851_c0_seq1 & 44903417 & 32.47 & 77 & 5-alpha-taxadienol-10-beta-hydroxylase \\
\hline comp110423_c0_seq1 & 60459952 & 41.38 & 87 & taxane 13-alpha-hydroxylase \\
\hline comp69534_c0_seq1 & 60459952 & 33.79 & 441 & taxane 13-alpha-hydroxylase \\
\hline comp38773_c1_seq1 & 60459952 & 45.83 & 96 & taxane 13-alpha-hydroxylase \\
\hline comp36415_c0_seq1 & 60459952 & 34.19 & 427 & taxane 13-alpha-hydroxylase \\
\hline comp57975_c1_seq1 & 60459952 & 44.93 & 69 & taxane 13-alpha-hydroxylase \\
\hline comp104139_c0_seq1 & 60459952 & 42.17 & 83 & taxane 13-alpha-hydroxylase \\
\hline comp93979_c0_seq1 & 75297723 & 38.03 & 71 & Taxane 14b-hydroxylase \\
\hline comp61533_c1_seq1 & 380039801 & 33.57 & 143 & taxane 14b-hydroxylase \\
\hline comp143394_c0_seq1 & 380039801 & 29.17 & 120 & taxane 14b-hydroxylase \\
\hline comp74596_c0_seq1 & 380039801 & 29.51 & 122 & taxane 14b-hydroxylase \\
\hline comp84707_c0_seq1 & 380039801 & 35.22 & 230 & taxane 14b-hydroxylase \\
\hline comp36896_c0_seq1 & 67633430 & 30.84 & 467 & taxoid 2-alpha-hydroxylase \\
\hline comp192945_c0_seq1 & 238915468 & 43.75 & 64 & taxoid 7-beta-hydroxylase \\
\hline comp36946_c0_seq1 & 365776087 & 55 & 60 & transcription factor WRKY \\
\hline comp64330_c0_seq1 & 365776087 & 54.24 & 59 & transcription factor WRKY \\
\hline comp78449_c0_seq1 & 365776087 & 66.67 & 54 & transcription factor WRKY \\
\hline comp67132_c0_seq1 & 365776087 & 56.34 & 71 & transcription factor WRKY \\
\hline comp59687_c0_seq1 & 365776087 & 41.22 & 131 & transcription factor WRKY \\
\hline comp68275_c0_seq1 & 365776087 & 56.72 & 67 & transcription factor WRKY \\
\hline comp104123_c0_seq1 & 222355764 & 29.87 & 154 & JAMYC \\
\hline comp69212_c0_seq1 & 222355764 & 41.69 & 710 & JAMYC \\
\hline comp69212_c0_seq2 & 222355764 & 46.72 & 259 & JAMYC \\
\hline comp69212_c0_seq3 & 222355764 & 41.69 & 710 & JAMYC \\
\hline
\end{tabular}


Table 2 ESTs homologous to genes involved in taxol synthesis in Taxus (Continued)

\begin{tabular}{llccc}
\hline comp124731_c0_seq1 & 222355764 & 100 & 27 & JAMYC \\
comp69971_c3_seq1 & 222355764 & 29.9 & 204 & JAMYC \\
comp38183_c0_seq2 & 222355764 & 30 & 160 & JAMYC \\
comp83061_c0_seq1 & 222355764 & 35.71 & 112 & JAMYC \\
\hline
\end{tabular}

Protein Gls, instead of their accession numbers, are provided here for convenience in table layout. These can be queried at NCBI protein databases.

identities (98.39\%, 98.45\% and 99.2\%) to the corresponding taxol genes in yew species (Table 3). Undoubtedly, these unsolved questions merited further study, especially from genomic scanning and experimental tests.

\section{Conclusions}

In the present study, the transcriptome of $C$. mandshurica was de novo assembled with Trinity and functionally annotated with Blast2go and KAAS. We found that highly differentiated genes between C. mandshurica and C. avellana correlated with local adaptation of the two species. In addition, a set of genes that might contribute to taxol production were identified and genetic mechanisms for taxol synthesis in distantly related plants were discussed. Thus, our study broadened the available transcriptome resources for Corylus, and provided meaningful information for researchers interested in taxol synthesis and high tolerance of $C$. mandshurica to fungal infection and cold stress.

\section{Methods}

\section{Sequencing and assembly}

Total RNA was extracted from leaves of C. mandshurica according to the CTAB protocol. The integrity of RNA was detected on an Agilent 2100 Bioanalyzer. The initial $20 \mu \mathrm{g}$ of total RNA was purified using polydT conjugated beads to extract polyA-tagged mRNA, which was subsequently cleaved into $\sim 200 \mathrm{bp}$ fragments by treatment with divalent cations at $75^{\circ} \mathrm{C}$. The first strand cDNA synthesis was carried out using reverse transcriptase (Invitrogen) with random hexamer primers, and the second strand using RNase $\mathrm{H}$ (Invitrogen)

Table 3 Proteins most homologous to genes involved in taxol synthesis in species outside Taxus

\begin{tabular}{|c|c|c|c|c|c|c|}
\hline Query Protein GI & Hit Protein GI & Identity (\%) & Taxonomy & Hit Protein GI & Identity (\%) & Taxonomy \\
\hline 15080743 & - & - & - & 62511183 & 48.53 & Abies grandis \\
\hline 38201489 & - & - & - & 62511183 & 48.66 & Abies grandis \\
\hline 386304920 & - & - & - & 62511183 & 49.3 & Abies grandis \\
\hline 24266823 & 56609042 & 97.99 & Ozonium sp. BT2 & 75319884 & 43.97 & Picea sitchensis \\
\hline 44903417 & 56609042 & 99.2 & Ozonium sp. BT2 & 75319884 & 44.17 & Picea sitchensis \\
\hline 53759170 & 56609042 & 66.38 & Ozonium sp. BT2 & 75319884 & 44.44 & Picea sitchensis \\
\hline 60459952 & 56609042 & 63.9 & Ozonium sp. BT2 & 75319884 & 45.32 & Picea sitchensis \\
\hline 67633430 & 56609042 & 56.34 & Ozonium sp. BT2 & 75319884 & 40.89 & Picea sitchensis \\
\hline 75297723 & 56609042 & 60.72 & Ozonium sp. BT2 & 75319884 & 42.5 & Picea sitchensis \\
\hline 238915468 & 56609042 & 56.2 & Ozonium sp. BT2 & 75319884 & 40 & Picea sitchensis \\
\hline 380039801 & 56609042 & 61.12 & Ozonium sp. BT2 & 75319884 & 42.92 & Picea sitchensis \\
\hline 386304485 & 56609042 & 66.22 & Ozonium sp. BT2 & 75319884 & 47.11 & Picea sitchensis \\
\hline 28380187 & 62461771 & 98.39 & fungal sp. BT2* & 148906373 & 43.98 & Picea sitchensis \\
\hline 28558088 & 62461771 & 60.23 & fungal sp. BT2 & 148906373 & 45.62 & Picea sitchensis \\
\hline 53690152 & 62461771 & 60.83 & fungal sp. BT2 & 148906373 & 44.25 & Picea sitchensis \\
\hline 339521621 & 62461771 & 60 & fungal sp. BT2 & 148906373 & 39.83 & Picea sitchensis \\
\hline 386304662 & 62461771 & 98.23 & fungal sp. BT2 & 148906373 & 44.07 & Picea sitchensis \\
\hline 386304248 & 169135276 & 98.45 & Cladosporium cladosporioides & 148906373 & 44.64 & Picea sitchensis \\
\hline 365776087 & - & - & - & 167859869 & 43.18 & Picea abies \\
\hline 222355764 & - & - & - & 148906957 & 46.99 & Picea sitchensis \\
\hline
\end{tabular}

\footnotetext{
* Ozonium sp. BT2 and fungal sp. BT2 are the same fungus species. [49,51].
}

Columns 2-4 show top hit protein information from fungi; columns 5-7 show top hit protein information from plants. Methodically, protein queries are blasted against NCBI nonredundant protein database and protein hits from the two designated sources with top sequence identity are recorded. Protein Gls, instead of their accession numbers, are provided here for convenience in table layout. These can be queried at NCBI protein databases. The reason for only three identified hit proteins from fungi is possibly due to the absence of genome data for taxol-producing fungi. 
and DNA polymerase I (New England BioLabs). Sequencing was performed on an Illumina Genome Analyzer II.

After removal of adapter sequences, raw reads were filtered according to stringent criteria [52]. The clean reads generated were used for all subsequent analyses. Trinity was used to assemble the paired-end short reads into contigs.

\section{Functional annotation}

The EST sequences were searched against the NCBI non redundant protein database using BLASTX with an E-value cutoff of 1e-5. The blast output in XML format was then annotated by Blast2go using default parameters. Kyoto Encyclopedia of Genes and Genomes (KEGG) was a universally acknowledged database for delineating networks of macromolecular interaction within cells. Pathway annotation was conducted using the KEGG Automatic Annotation Server (KAAS) web server and Single-directional Best Hit ( $\mathrm{SBH})$ method against representative sets for eukaryotes. GO enrichment was analyzed with WEGO.

\section{Ortholog identification and comparison}

Bi-directional BLASTN searches were performed for the transcriptomes of C. mandshurica and C. avellana. The reciprocal best blast hits were considered as orthologs. Orthologs with sequence identity lower than 90\% were discarded in further GO analyses in order to exclude distant homologs due to the incomplete and fragmentary nature of transcriptomes. Two types of sequence variations were studied in GO enrichment analyses. One type focuses on orthologs with relatively low sequence identity, which includes $10.4 \%$ of all orthologs with sequence identity less than $97 \%$. The other focuses the presence of gaps in local alignments of orthologs as shown in BLASTN outputs. GO terms of all orthologs and these two types of orthologs were extracted from Blast2go outputs. GO enrichment analyses were carried out on WEGO server. GO terms with p-value of Pearson Chi-square test below 0.05 was considered statistically significant.

\section{The identification of genes involved in taxol synthesis}

Genes related to taxol syntheses were identified by extensively parsing gene descriptions in the XML-formatted BLASTX output using key words of all the corresponding enzymes. The potential genes were further manually verified.

In order to compare these sequences with homologous genes in other species, we used the prototype genes responsible for taxol synthesis in yew as query sequences to search against the NCBI non redundant protein database using BLASTP. The top protein hits from fungi and plants were extracted.
DATA Availability

Reads are deposited at NCBI SRA (SRR857924).

\section{Additional files}

Additional file 1: The assembled EST sequences of $C$. mandshurica in fasta format.

Additional file 2: GO terms for orthologs with sequence identity lower than 97.

Additional file 3: GO terms for orthologs with INDEL.

Additional file 4: GO terms for all orthologs.

Competing interests

The authors declare that they have no competing interests.

Authors' contributions

$H M$ and $J L$ analyzed the data and wrote the manuscript. $Z L$ acquired the leaf sample. BL prepared the mRNA for sequencing. QQ provided helpful suggestion in data analysis. All authors read and approved the final manuscript.

\section{Acknowledgements}

This study is supported by the National High Technology Research and Development Program of China (863 Program, No. 2013AA100605), Research Fund for the Doctoral Program of Higher Education of China (Grant No. 20100211110008) and the Fundamental Research Funds for the Central Universities (lzujbky-2009-k05).

Received: 10 June 2013 Accepted: 2 October 2013

Published: 5 October 2013

\section{References}

1. Zhang Y, Li F, Tao R, Li Z, Liang Y: An investigation of wild Corylus resource at Changbai Mountains. J Jilin Agri Sci 2007, 32(5):56-57.

2. Liu H: Exploring the utilization of Corylus. Farm Prod Proc 2010, 1:24-25.

3. Huang M: Selecting for excellent clones of Castanea henryi. Fujian Agri Sci Technol 2012, 12:35-40.

4. Liu Y, Zhang H, Zhang W: The new application of Corylus. Spel Econ Anim Plant 1998, 6:38.

5. Plosker GL, Hurst M: Paclitaxel: a pharmacoeconomic review of its use in non-small cell lung cancer. Pharmacoeconomics 2001, 19(11):1111-1134.

6. Kumar S, Mahdi H, Bryant C, Shah JP, Garg G, Munkarah A: Clinical trials and progress with paclitaxel in ovarian cancer. Inter J Women's Health 2010, 2:411-427.

7. Gradishar WJ: Taxanes for the treatment of metastatic breast cancer. Bre Can: Basic Clin Res 2012, 6:159-171.

8. Wani MC, Taylor HL, Wall ME, Coggon P, MCPhail AT: Plant antitumor agents. VI. Isolation and structure of taxol, a novel antileukemic and antitumor agent from Taxus brevifolia. J Am Chem Soc 1971, 93(9):2325-2327.

9. Vidensek N, Lim P, Campbell A, Carlson C: Taxol content in bark, wood, root, leaf, twig, and seedling from several Taxus species. J Nat Prod 1990, 53(6):1609-1610.

10. Service RF: Hazel trees offer new source of cancer drug. Science 2000, 288(5463):27-28.

11. Bestoso F, Ottaggio L, Armirotti A, Balbi A, Damonte G, Degan P, Mazzei M, Cavalli F, Ledda B, Miele M: In vitro cell cultures obtained from different explants of Corylus avellana produce Taxol and taxanes. BMC Biotechnol 2006, 6(1):45.

12. Hoffman A, Shahidi F: Paclitaxel and other taxanes in hazelnut. $J$ Funct Foods 2009, 1(1):33-37.

13. Ottaggio L, Bestoso F, Armirotti A, Balbi A, Damonte G, Mazzei M, Sancandi M, Miele M: Taxanes from Shells and Leaves of Corylus avellana. J Nat Prod 2007, 71(1):58-60.

14. Luo F, Fei $X$, Tang F, Li X: Simultaneous determination of Paclitaxel in hazelnut by HPLC-MS/MS. For Res 2011, 24(6):779-783.

15. Miele M, Mumot A, Zappa A, Romano P, Ottaggio L: Hazel and other sources of paclitaxel and related compounds. Phytochem Rev 2012, 11(2-3):211-225. 
16. Liu X-K, Liu J-J: New source for L-iditol and taxanes. Nat Preced 2008. http://precedings.nature.com/documents/1502/version/1.

17. Bemani E, Ghanati F, Rezaei A, Jamshidi M: Effect of phenylalanine on taxol production and antioxidant activity of extracts of suspensioncultured hazel (Corylus avellana L.) cells. J Nat Med 2013, 67(3):446-451.

18. Zhao D, Su S, Ni B, Wang W, Meng X, Liu W: Germplasm resources investigation and utilization prospects of hazel in Small Xing'an Ridge region. Chin Agric Sci Bull 2012, 28(28):87-94

19. Coyne CJ, Mehlenbacher SA, Smith DC: Sources of resistance to Eastern Filbert Blight in hazelnut. J Am Soc Hortic Sci 1998, 123(2):253-257.

20. Molnar TJ, Capik J, Zhao S, Zhang N: First report of Eastern Filbert Blight on Corylus avellana 'Gasaway' and 'VR20-11' caused by Anisogramma anomala in New Jersey. Plant Dis 2010, 94(10):1265-1265.

21. Liang W: An investigation of wild Corylus resources in China. Journal of Liaoning Forestry Science and Technology 1989, 1:45-52

22. Peng L, Wang $M$, Liang W, Xie M, Li D: A study on cold resistance for filbert genus (Corylus L.) plants. J Jilin Fores Univ 1994, 3:166-170.

23. Ni B, Ni W, Xu X, Wang X: Hazel breeding research. Forest By-Product and Speciality in China 2010, 106(3):29-31.

24. $X-j$ Z, F-x D, R-q Z, G-x W, M-p Y, L-s L: Research on the compatibility of five Corylus species. J Cen South Univ Fores Technol 2009, 29(4):26-30.

25. Erdogan $V$, Mehlenbacher SA: Interspecific hybridization in hazelnut (Corylus). J Am Soc Hortic Sci 2000, 125(4):489-497.

26. Liang $W$, Xie $M$, Dong $D$, Jiang Z: The breeding research for new Corylus cultivar. China Fruits 2000, 2:4-6.

27. Cheng L, Huang W, Zhou Z, Liu J, Wang Y, Su S, Zhai M: Genetic diversity of six Corylus species in China detected with microsatellite isolated from Corylus avellana. Scientia Silvae Sinicae 2009, 45(2):22-26.

28. Li X, Li X, Wang Z, Xue C, Limin Z, Guo Y: Study on phylogenetic analysis of Corylus germplasm resouces with SSR molecular markers for Corylus avellana. J Northeast Agric Univ 2011, 42(4):129-136.

29. Rowley ER, Fox SE, Bryant DW, Sullivan CM, Priest HD, Givan SA, Mehlenbacher SA, Mockler TC: Assembly and characterization of the European hazelnut 'Jefferson' transcriptome. Crop Sci 2012, 52(6):2679-2686

30. Grabherr MG, Haas BJ, Yassour M, Levin JZ, Thompson DA, Amit I, Adiconis $X$, Fan L, Raychowdhury R, Zeng Q, et al: Full-length transcriptome assembly from RNA-Seq data without a reference genome. Nat Biotechnol 2011, 29(7):644-652.

31. Min XJ, Butler G, Storms R, Tsang A: OrfPredictor: predicting protein-coding regions in EST-derived sequences. Nucleic Acids Res 2005, 33(suppl 2):W677-W680.

32. Wang N, Thomson M, Bodles WJA, Crawford RMM, Hunt HV, Featherstone AW, Pellicer J, Buggs RJA: Genome sequence of dwarf birch (Betula nana) and cross-species RAD markers. Mol Ecol 2013, 22(11):3098-3111.

33. Kent WJ: BLAT-The BLAST-Like Alignment Tool. Genome Res 2002, 12(4):656-664.

34. Altschul SF, Madden TL, Schäffer AA, Zhang J, Zhang Z, Miller W, Lipman DJ: Gapped BLAST and PSI-BLAST: a new generation of protein database search programs. Nucleic Acids Res 1997, 25(17):3389-3402.

35. Conesa A, Götz S, García-Gómez JM, Terol J, Talón M, Robles M: Blast2GO: a universal tool for annotation, visualization and analysis in functional genomics research. Bioinformatics 2005, 21(18):3674-3676.

36. Moriya Y, Itoh M, Okuda S, Yoshizawa AC, Kanehisa M: KAAS: an automatic genome annotation and pathway reconstruction server. Nucleic Acids Res 2007, 35(suppl 2):W182-W185.

37. Srivastava A, Cai L, Mrázek J, Malmberg RL: Mutational patterns in RNA secondary structure evolution examined in three RNA families. PLOS ONE 2011, 6(6):e20484.

38. Pelletier J, Sonenberg $\mathrm{N}$ : Insertion mutagenesis to increase secondary structure within the $5^{\prime}$ noncoding region of a eukaryotic mRNA reduces translational efficiency. Cell 1985, 40(3):515-526.

39. Brown PH, Tiley LS, Cullen BR: Effect of RNA secondary structure on polyadenylation site selection. Genes Dev 1991, 5(7):1277-1284

40. McManus CJ, Graveley BR: RNA structure and the mechanisms of alternative splicing. Curr Opin Genet Dev 2011, 21(4):373-379.

41. Mauger DM, Siegfried NA, Weeks KM: The genetic code as expressed through relationships between mRNA structure and protein function. FEBS Lett 2013, 587(8):1180-1188.

42. Amini F, Ismail E: 3'-UTR variations and G6PD deficiency. J Hum Genet 2013, 58(4):189-194
43. Ye J, Fang L, Zheng H, Zhang Y, Chen J, Zhang Z, Wang J, Li S, Li R, Bolund L, et al: WEGO: a web tool for plotting GO annotations. Nucleic Acids Res 2006, 34(suppl 2):W293-W297.

44. Xiong Z, Yang $Y$, Zhao N, Wang Y: Diversity of endophytic fungi and screening of fungal paclitaxel producer from Anglojap yew. Taxus $x$ media. BMC Microbiol 2013, 13(1):71.

45. Li S, Zhang P, Zhang M, Fu C, Yu L: Functional analysis of a WRKY transcription factor involved in transcriptional activation of the DBAT gene in Taxus chinensis. Plant Biol 2013, 15(1):19-26.

46. Nims E, Vongpaseuth K, Roberts SC, Walker EL: WITHDRAWN: TcJAMYC: A bHLH transcription factor that activates paclitaxel biosynthetic pathway genes in yew (This manuscript was accepted by the Journal of Biological Chemistry, but following acceptance discrepancies in some of the sequences used in the work were discovered. The manuscript was withdrawn, and additional work has been conducted. Submission of a new manuscript is anticipated.). J Biol Chem 2009 http://www.jbc.org/ content/early/2009/10/01/jbc.M109.026195.

47. Sun G, Yang Y, Xie F, Wen J-F, Wu J, Wilson IW, Tang Q, Liu H, Qiu D: Deep sequencing reveals transcriptome re-programming of Taxus $\times$ media cells to the elicitation with methyl jasmonate. PLOS ONE 2013, 8(4):e62865.

48. Chen F, Tholl D, Bohlmann J, Pichersky E: The family of terpene synthases in plants: a mid-size family of genes for specialized metabolism that is highly diversified throughout the kingdom. Plant J 2011, 66(1):212-229.

49. Guo BH, Wang YC, Hu H, Miao ZQ, Tang KX: An endophytic Taxol-producing fungus BT2 isolated from Taxus chinensis var. maire. Afr J Biotechnol 2006, 5(10):875-877.

50. Zhang P, Zhou P-P, Yu L-J: An endophytic taxol-producing fungus from Taxus media, Cladosporium cladosporioides MD2. Curr Microbiol 2009, 59(3):227-232

51. Wei Y, Zhou X, Liu L, Lu J, Wang Z, Yu G, Hu L, Lin J, Sun X, Tang K: An efficient transformation system of taxol-producing endophytic fungus EFY-21 (Ozonium sp.). Afr J Biotechnol 2010, 9(12):1726-1733.

52. Qiu Q, Ma T, Hu Q, Liu B, Wu Y, Zhou H, Wang Q, Wang J, Liu J: Genome-scale transcriptome analysis of the desert poplar. Populus euphratica. Tree Physiol 2011, 31(4):452-461.

doi:10.1186/1471-2229-13-152

Cite this article as: Ma et al:: Transcriptome analyses of a Chinese hazelnut species Corylus mandshurica. BMC Plant Biology 2013 13:152.

\section{Submit your next manuscript to BioMed Central and take full advantage of:}

- Convenient online submission

- Thorough peer review

- No space constraints or color figure charges

- Immediate publication on acceptance

- Inclusion in PubMed, CAS, Scopus and Google Scholar

- Research which is freely available for redistribution 